\title{
Treatment of Complications of Spinal Cord Injuries
}

\author{
Jack Kushner* \\ Head Prof Med, MD, Annapolis, Maryland
}

Received: April 24, 2018; Published: May 07, 2018

*Corresponding author: Jack Kushner, Head Prof Med, MD, Annapolis, Maryland, Tel: 4107573754; Email: jkaoportal@comcast.net

\begin{abstract}
In addition to the challenges of treating patients with spinal cord injuries, it can be most distressing and daunting managing the complications with these injuries. This article discusses some of these problems and how to avoid and treat many of them.
\end{abstract}

Keywords: Spinal Cord injuries

\section{Mini Review}

In addition to the complications of spinal cord injuries, there are additional spinal cord injuries which are the result of spinal cord surgery. For example, some patients have presented with signs and symptoms of cervical spondylosis. This refers to severe cervical osteoarthritis resulting in spinal stenosis particularly in the cervical region. The patient complains of loss of fine movement of his fingers, weakness in his arms and legs, perhaps loss of pain and temperature on one or both sides of the body, and partial loss of his bowel and bladder functions. The plain cervical spine $\mathrm{x}$-rays will show huge osteophytes pressing on the cervical spinal cord at multiple levels and the CAT scan and MRI will confirm the compression and the spinal stenosis. For many years the preferred surgical treatment was a decompressive posterior cervical laminectomy at multiple levels and despite care with the positioning of the patient without any flexion of the neck, despite care not to insert the foot of the surgical rongeur under the lamina at any level, and regardless whether or not the dentate ligaments were sectioned, it was unfortunately not uncommon for the patient to awake from the surgery with his tetraparesis having progressed to a tetraplegia. This does not happen so frequently now since more surgeons are treating this condition with an anterior cervical decompression at multiple levels followed by a bony fusion with cervical plates and screws.

One of the more devastating and frustrating complications of the spinal cord injury is that of chronic pain. It is discouraging enough for a patient to be paralyzed, but to also suffer from chronic pain for which there is no satisfactory treatment makes the whole situation even worse. This can lead and does lead to depression and often to suicide. Although psychiatric consultation might be helpful, the treating neurosurgeon has to actively attempt to control this problem. The International Association for the Study of Pain has defined two distinct categories of pain [1]. These two types are classified as at-level neuropathic pain and below-level neuropathic pain. According to Tator, Fehlings, and Yezierski the at-level neuropathic pain is associated with the damage to the gray matter above and below the injury site, and the amount of cellular damage caused by the release of neurotransmitters such as glutamate and $\mathrm{N}$-methyl-D-aspartate or NMDA [2,3]. Bowsher has shown that below-level neuropathic pain is caused by loss of axonal function, particularly the spinothalamic tract [4]. Neuropathic pain is caused by damage to the neural tissue and nociceptive pain is caused by damage to the bone, muscles, and ligaments. Nociceptive pain can be managed by the usual pain medications and anti-inflammatory drugs and analgesics. Neuropathic pain can be treated with anticonvulsants and antidepressant medications, but not very well.

Another complication of spinal cord injuries is that of spasticity. This term refers to the muscle spasms and increased muscle tone seen after a spinal cord injury. Whereas the pain is due to the increased activities in the pain pathways, the spasms are due to the increased activity of the reflex pathways such as proprioception neurons and the motor neurons. The fact that various and numerous treatments are tried is a testament to the fact that no one treatment is uniformly effective in all patients. For example, rehabilitation and stretching techniques can be helpful and if not, drug therapy can be attempted. If this spasticity is severe, then surgery is recommended and baclofen with an implanted pump can be given [5]. Baclofen and Tizanidine have inhibitory effects on the motor neurons. Some have had success with Fampridine which is a potassium channel 
blocker. A side effect of these drugs may be that they interfere with a patient's own muscle contraction [6].

The incidence of deep vein thrombosis (DVT) and pulmonary embolism resulting from the immobility of patients with spinal cord injuries have dramatically decreased due to increased awareness of the problem and prophylactic measures which are now the standard of care. If in fact, they do occur it is a life threatening complication. DVTs are blood clots that form in the deep veins of the legs and thighs and can result in blood clot lodging in the pulmonary artery blocking the flow of oxygenated blood to the remainder of the body. Preventive regimens have decreased thromboembolism from $14 \%$ to $9.8 \%$ and the incidence of embolism from $4 \%$ to $2.6 \%$ [7]. The standard of care for preventing thromboembolism requires the treatment with low-molecular-weight Heparins or adjusted dose Heparin and the use of rotating beds. A combination of compression stockings and electrical stimulation can also be used, but high doses of Heparin may cause bleeding [8].

Urinary bladder dysfunction occurs in all patients with severe spinal cord injuries and there are three different types of bladder problems. The flaccid bladder occurs when there is an injury to the sacral spinal cord, which causes the bladder's detrusor muscle to be incapable of contracting. In order to prevent the urine from backing up into the kidneys, intermittent catherterization is ordered. The other types of bladder dysfunction are detrusor hyperreflexia and detrusor-spincter dyssynergia. The bladder hyperreflexia is treated to avoid urinary incontinence and treatment of the dyssynergia ensures adequate drainage. Both of these conditions are treated with anti cholinergic medications to suppress the contractions. If these medications are ineffective, then an augmentation cystoplasty is suggested for those who have detrusor hyperreflexia [9]. There are other medications and surgical procedures for the treatment of these bladder dysfuntion problems, but as of yet these need to be investigated more thoroughly prior to being listed with evidenced based medical remedies.

Most patients with spinal cord injuries do not have voluntary control of stool elimination. This problem is considered by most of the patients to be one of the life- limiting conundrums. Prior to discharge from the hospital or rehabilitation center, spinal cord injury patients are taught how to manage the neurogenic bowel. Proper care in necessary so as to have regular bowel movements and to prevent constipation, incontinence, and bowel obstruction from impacted bowels. Treatment usually includes the administration of dietary fiber, adequate fluid intake, oral medications to loosen the stools, and rectal suppositories. Since some patients have a reflexive bowel and others have an a reflexive bowel, the difference in treatment comes down to the type of rectal stimulant and the consistency of the stools. The Spinal Cord Medicine Consortium has issued practice guidelines for the management of the neurogenic bowel [10].

Patients with a spinal cord injury above T6 can develop a potentially lethal complication called autonomic dysreflexia, which is manifested by severe headache, hypertension, and profuse sweating. These symptoms result from an over activation of the autonomic nervous system in the spinal cord presumably because of blocked nervous impulses from the brain, which usually restrain these cells. The previously described problems with the urinary bladder and the bowel can trigger this reaction. Thus, the overactive sympathetic nervous system causes a narrowing of the blood vessels which elevates the blood pressure and can cause the patient to have seizures, a stroke, or cardiac arrhythmia. Many patients learn to care for these emergencies by sitting up and letting the bladder and bowel drain. Otherwise, there are numerous medications for this medical problem [11].

Pressure ulcers unfortunately frequently occur with these patients and can be serious. By and large these ulcers can be avoided if proper care is rendered to these spinal cord injured patients. They are caused by constant pressure on the tissue surface. This pressure affects the capillaries and the resulting ischemia causes ulceration, which can be minor such as skin discoloration or can be a Grade IV resulting in necrosis of the skin, muscle, bone, and surrounding tissues. The Consortium for Spinal Cord Medicine has issued practical clinical guideless on how to avoid and also how to treat these ulcerations. Their recommendation is that care be taken to avoid prolonged positional immobilization and to use support devices on beds and wheelchairs. Treatment may be as simple as dietary changes and local tissue treatment or it may be more extensive requiring surgical debridement for the stage III and IV ulcerations. Electrical stimulation may also be used and can be helpful [12].

Treatment research for male sexual dysfunction and fertility has focused mainly on erectile dysfunction rather than on ejaculation and orgasm. The primary therapy for this problem has been the administration of oral Viagra. With this drug most male patients are able to have an erection, but all patients are not able to maintain the erection sufficiently to sustain intercourse. Fertility problems usually result from poor sperm quality and ejaculatory dysfunction. Penile vibratory stimulation has become routine for those men who wish to have children. Studies show that approximately one third of couples are able to achieve pregnancy using this vibratory stimulation. Even this is dependent on overcoming the poor sperm quality in men and may require artificial insemination, in vitro fertilization, or intracytoplasmic sperm injections [13].

The problems with female sexual dysfunction and fertility in those patients with spinal cord injuries include insufficient vaginal secretion and failure to reach orgasm. Women also benefit from Viagra as this promotes increased sexual arousal and works well with manual or visual stimulation. Since fertility in women is not dependent on spinal circuits, women are able to have babies, but these pregnancies can lead to numerous potential complications.

Because of the decreased mobility of the spinal cord injured patient, there is a decrease in bone density and an increase in the formation of heterotopic ossification. The loss of bone density can 
lead to an increase in bone fractures and can be treated with various medications. One of the most popular medications is Fosamax, but some patients have difficulty taking this due to gastrointestinal discomfort. Heterotopic ossification may just be a mild x-ray finding or it can be a massive bone growth near the hips and may require surgical extirpation. Non-steroidal anti-inflammatory medications have been helpful in preventing this extra bone formation especially when used with range of motion exercises.

As one can imagine, depression is a real problem in patients with spinal cord injuries and for good reason. The American Psychiatric Association has given some specific guidelines for the treatment of this problem [14]. Although this discussion has mainly been about the use of pharmacological agents and surgery as the main therapeutic avenues, there have been great strides in the use of physical training and rehabilitation techniques as additional tools to help patients with a spinal cord injury. In addition, neuroprostheses can be utilized as aids to help these patients. Body weight support techniques can be used to assist in locomotion. For example, in order to help some of these patients try to walk again, they can work with body weight-supported treadmill training. These patients are placed in a harness to unload up to $50 \%$ of their weight and then put on a treadmill to simulate walking. This is promising but more needs to be done to see whether or not this form of therapy has any value. In combination with this is the use of functional electrical stimulation especially in incomplete spinal cord injuries. This can be used in multiple ways such as cardiovascular conditioning, improving gait control, restoring hand control, breathing, and bowel and bladder function such as with Vocare. The FDA has approved a neuroprosthesis for hand control called Freehand. Phrenic nerve pacing might be helpful for those patients who have problems breathing. In addition, the FDA has also approved a functional electrical system for ambulation which is a surface stimulation system called Parastep.

\section{References}

1. Vierck CJ Jr, Siddall P, Yezierski RP (2000) Pain following spinal cord injury: Animal models and mechanistic studies. Pain 89(1): 1-5.
2. Tator CH, Fehlings MG (1991) Review of the secondary injury theory of acute spinal cord trauma with emphasis on vascular mechanisms. Journal of Neurosurgery 75(1): 15-26.

3. Yezierski RP (2000) Pain following spinal cord injury: Pathophysiology and central mechanisms. Progress in Brain Research 129: 429-449.

4. Bowsher D (1996) Central pain: Clinical and physiological characteristics. Journal of Neurology, Neurosurgery, \& Psychiatry 61(1): 19-28.

5. Kirshblum S (2004) New rehabilitation interventions in spinal cord injury. Journal of Spinal Cord Medicine 27(4): 342-350.

6. McDonald JW, Becker D (2003) Spinal cord injury: Promising interventions and realistic goals. American Journal of Physical Medicine and Rehabilitation 82(10): S38-49.

7. Chen D, Apple DF Jr, Hudson LM, Bode R (1999) Medical complications during acute rehabilitation following spinal cord injury-Current experience of the model systems. Archives of Physical Medicine and Rehabilitation 80(11): 1397-1401.

8. Hadley MN, Walters BC (2002) Deep venous thrombosis and thromboembolism in patients with cervical spinal cord injuries. Neurosurgery 50(3): S73-80.

9. Sidi AA, Becher EF, Reddy PK, Dykstra DD (1990) Augmentation enterocystoplasty for the management of voiding dysfunction in spinal cord patients. Journal of Urology 143(1): 83-85.

10. Spinal Cord Medicine Consortium (1998) Clinical practice guidelines: Neurogenic bowel management in adults with spinal cord injury. Journal of Spinal Cord Medicine 21(3): 248-293.

11. Consortium for Spinal Cord Medicine (2001) Acute Management of Autonomic Dysreflexia: Individuals with Spinal Cord Injury Presenting to Health-Care Facilities. Paralyzed Veterans of America 25(1): S67-88.

12. Consortium for Spinal Cord Medicine (2000) Pressure Ulcer Prevention and Treatment Following Spinal Cord Injury: A Clinical Practice Guideline for Healthcare Professionals. Paralyzed Veterans of America, USA 24(1): 40-101

13. Sonksen J, Sommer P, Biering Sorensen F, Ziebe S, Lindhard A, et al. (1997) Pregnancy after assisted ejaculation procedures in men with spinal cord injury. Archives of Physical Medicine and Rehabilitation 78(10): 1059-1061.

14. Carl C Bell (1994) Diagnostic and Statistical Manual of Mental Disorders: DSM-IV. American Psychiatric Association, USA 272(10): 828-829.

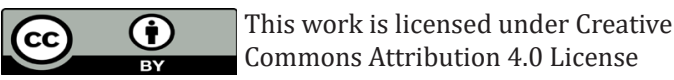

To Submit Your Article Click Here: Submit Article
DOI: 10.32474 /OAJBEB.2018.02.000137

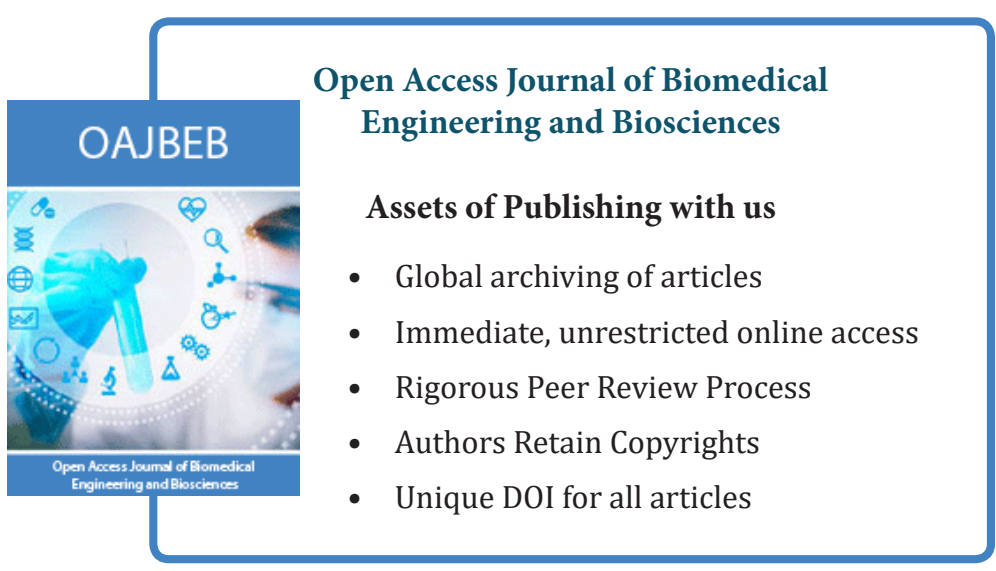

